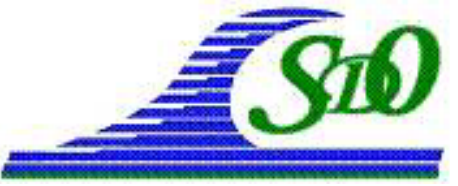

XI ${ }^{\text {èmes }}$ Journées Nationales Génie Côtier - Génie Civil

Les Sables d'Olonne, 22-25 juin 2010

DOI:10.5150/jngcgc.2010.042-M C Editions Paralia CFL

disponible en ligne - http://www.paralia.fr - available online

\title{
L'apport de modèles numériques pour l'étude morphodynamique d'un système dune-plage macrotidal sous l'effet des tempêtes : plage de la dune Dewulf, Est de Dunkerque, France
}

\section{Aurélie MASPATAUD ${ }^{1,2,3}$, Déborah IDIER ${ }^{4}$, Philippe LARROUDE ${ }^{5}$, François SABATIER ${ }^{6}$, Marie-Hélène RUZ ${ }^{1,2,3}$, Elodie CHARLES ${ }^{4}$, Sophie LECACHEUX ${ }^{4}$, Arnaud HEQUETTE ${ }^{1,2,3}$}

1. Univ Lille Nord de France, F-59000 Lille, France.

2. ULCO, LOG, F-62930 Wimereux, France.

3. CNRS, UMR8187, R-62930 Wimereux, France.Aurelie.Maspataud@univ-littoral.fr

4. BRGM, F-45060 Orléans, France.

5. LEGI, BP 53, 38041 Grenoble, France.

6. Aix Marseille université, CEREGE, BP80, 13545 Aix-en-Provence cedex04, France.

\section{Résumé :}

La mise en œuvre de 3 modèles numériques (SBEACH, X-BEACH, TELEMAC) sur une portion du littoral Est-dunkerquois, fragilisée par l'érosion pendant les évènements de tempête, a pour objectif d'évaluer la réponse de la morphodynamique du profil crossshore dune-plage sous l'effet d'évènements de forte énergie. La validation et la comparaison des résultats de ces codes morphodynamiques ont été effectuées à partir de mesures hydrodynamiques in-situ et de levés topographiques réalisés par temps calme ainsi qu'avant et après un évènement de tempête. Cette étude met ainsi en évidence la complémentarité des 3 modèles utilisés et la nécessité de continuer à les améliorer.

\section{Mots-clés :}

Modélisation - SBEACH - X-BEACH - TELEMAC - Tempête - Erosion Macrotidal - Morphodynamique

\begin{abstract}
:
The implementation of 3 numerical models (SBEACH, X-BEACH, TELEMAC) on a coastal stretch East of Dunkerque harbour, weakened by erosion during storm events, aims at evaluate the morphodynamic response of cross-shore dune-beach profile affected by strong energy events. The validation and the comparison of the results obtained with these morphodynamic models were carried out from in-situ hydrodynamic measurements and topographical surveys, for a period of calm weather and before/after storm event. This study highlights the complementarity of the 3 models and the need for their future improvement.
\end{abstract}

Keywords:

Modelling - SBEACH - X-BEACH - TELEMAC - Storm event - Erosion Macrotidal - Morphodynamic 


\section{Introduction}

Aujourd'hui, plus de $40 \%$ des littoraux métropolitains français sont en érosion, un phénomène au cœur des préoccupations environnementales. Dans le contexte actuel de changement climatique, il est devenu primordial d'étudier le comportement des plages afin de mieux comprendre leur fonctionnement, notamment à l'aide de modèles numériques qui permettent de simuler leur réponse morphodynamique aux forçages hydro-météorologiques. Cette étude, liée à la problématique du recul du trait de côte sous l'effet des tempêtes, porte sur la mise en œuvre de plusieurs modèles numériques sur un site côtier macrotidal afin d'évaluer la réponse de la morphodynamique du profil côtier sous l'effet d'événements de forte énergie. L'objectif principal est d'évaluer dans quelle mesure des modèles morphodynamiques seraient capables de reproduire la dynamique d'une portion du littoral fragilisée par l'érosion pendant les évènements de tempête. Ce travail s'inscrit dans le projet VULSACO dont l'objectif est de développer et d'estimer, pour 1'horizon 2030, des indicateurs de vulnérabilité physique des plages sableuses face aux phénomènes d'érosion et de submersion marine.

\section{Présentation du site d'étude}

Le site d'étude retenu est la plage de la Dune Dewulf, située à l'est de Dunkerque, dans le nord de la France. Comme toutes les plages macrotidales de la côte d'Opale (marnage $>5 \mathrm{~m}$ ), la plage de la Dune Dewulf est caractérisée par un large estran de sable fin et un système de barres et de bâches intertidales découvrant largement à marée basse (400-450 m en période de vives eaux). La morphodynamique de plage est ici dominée à la fois par les vagues, la marée et les courants de marée.

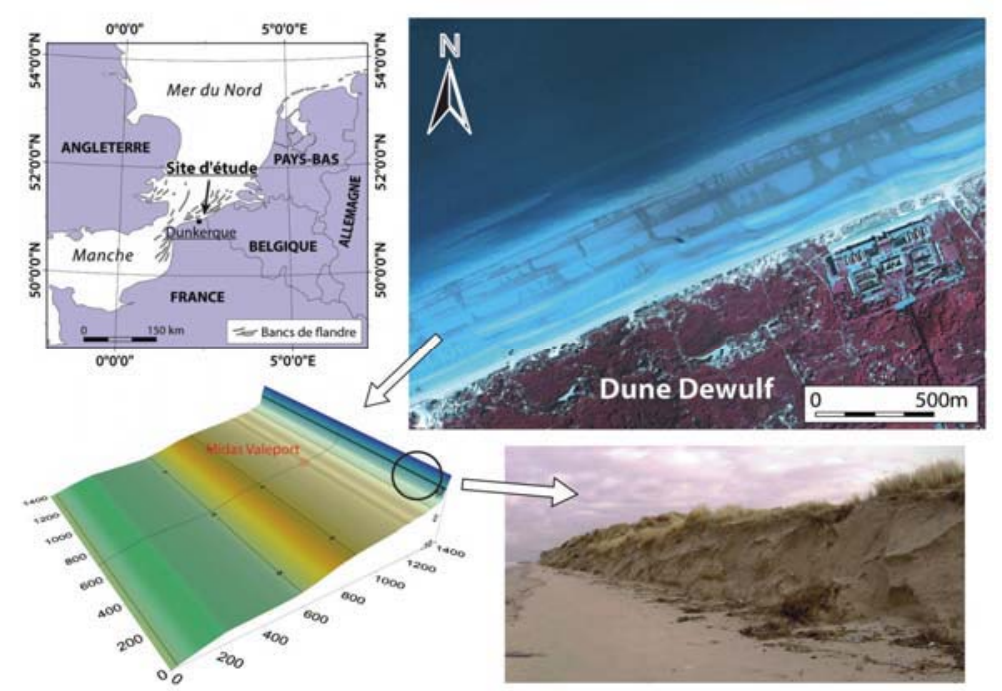

Figure 1. Localisation de la zone d'étude en érosion sur l'est-dunkerquois : orthophotographie littorale (IGN, 2000) et emprise spatiale de la topographie/bathymétrie. 
La plage est bordée par un cordon dunaire, de 5 à $16 \mathrm{~m}$ de hauteur, dont le versant externe est épisodiquement sapé en falaise vive et peut ainsi reculer de plusieurs mètres lors d'évènements de tempête (RUZ et al., 2009). A l'avant côte, les fonds sont marqués par la présence de nombreux bancs sableux (bancs de Flandre), très développés et orientés parallèlement à la côte, dont la dynamique est fortement liée à l'action des courants de marée. Le banc Hills, un important banc sableux situé à 2-3 km au large de la plage étudiée, est sub-affleurant à marée basse, ce qui peut avoir un impact sur l'hydrodynamique côtière (HEQUETTE et al., 2009). Les effets des tempêtes le long de ce littoral, soumis habituellement à des vagues de faible énergie, sont déterminants dans l'évolution du trait de côte.

\section{Modèles numériques et conditions de simulation}

\subsection{Présentation des trois codes morphodynamiques de modélisation court-terme}

(1) Le modèle cross-shore semi-empirique SBEACH est un outil numérique 2DV (bidimensionnel vertical), développé pour modéliser l'érosion des dunes et du haut de plage lors des tempêtes. Conçu par le CERC (LARSON \& KRAUS, 1989), ce programme est basé sur des relations semi-empiriques de transport sédimentaire, qui calculent les transformations d'un profil cross-shore, en fonction des conditions de houle au large et du profil d'équilibre. Ce modèle, qui ne prend pas en compte les courants de marée, a déjà montré des résultats satisfaisants en domaine microtidal (MORELLATO et al., 2004 ; SABATIER, 2008).

(2) X-BEACH est un modèle hydrodynamique (courants, vagues, ondes infragravitaires, undertow, ...) et morphodynamique (partie immergée, pied de dune, ...), encore en développement, conçu pour modéliser l'impact des évènements climatiques extrêmes sur les dunes. Ce modèle du domaine public est actuellement développé par UNESCOIHE, TU Delft et DELTARES (ROELVINK et al., 2008). Ce modèle permet de résoudre les équations de propagation des vagues, de transport et de conservation du sédiment (évolution du fond). Il est particulièrement adapté pour les évènements de tempêtes (BAART et al., 2009).

(3) Le système 2DH TELEMAC est une chaîne de calcul développée par le LNHE (HERVOUET, 2007) permettant de faire différents calculs hydro-sédimentaires tels que la propagation de la houle avec le code Tomawac, l'intéraction des courants de marée et de houle avec le code Telemac 2D, le transport solide avec le code Sisyphe (VILLARET, 2004). Ces deux derniers codes sont en couplage "interne", ce qui permet une réactualisation des fonds à chaque pas de temps (LARROUDE, 2008).

\subsection{Données et conditions de simulation}

Dans un premier temps, le modèle de vagues SWAN (BOOIJ et al., 2004) a été utilisé sur toute la Manche pour pouvoir propager les vagues jusqu'à l'avant-côte, à partir de 
données de vagues du modèle de prévision WW3 développé au NOAA/NCEP (CHARLES, 2008). SWAN fournit ainsi les conditions aux limites pour les modèles morphodynamiques. Dans un deuxième temps, les caractéristiques des vagues extraites des simulations SWAN ont été imposées à la limite offshore du domaine des modèles $(-10 \mathrm{~m})$. Les résultats des simulations ont ensuite été comparés avec des mesures des capteurs et les relevés topographiques, afin de calibrer au mieux les paramètres des modèles (CHARLES, 2008).

Tableau 1. Données utilisées en entrée des 3 modèles.

\begin{tabular}{|c|c|c|}
\hline & $S B E A C H$ & $T E L E M A C$ \\
\hline $\begin{array}{l}\text { Topographie / } \\
\text { Bathymétrie }\end{array}$ & Profil $(X, Z) d u$ 7/11/2007 & Bathymétrie $(X, Y, Z) d u$ 7/11/2007 \\
\hline Niveau d'eau & $\begin{array}{l}\text { Variations du niveau } \\
\text { d'eau (m) }\end{array}$ & $\begin{array}{l}\text { Variations du niveau d'eau (m), avec un déphasage } \\
\text { entre les limites Est et Ouest du domaine de calcul }\end{array}$ \\
\hline Vagues & \multicolumn{2}{|c|}{$\begin{array}{l}\text { Hs (m), Tpic (s), Direction }\left(^{\circ}\right) \text {, dans le système de coordonnées spécifique à } \\
\text { chaque modèle) }\end{array}$} \\
\hline Vent & Intensité et direction & - \\
\hline
\end{tabular}

Le domaine de calcul choisi pour X-BEACH et TELEMAC est un domaine 2D de 1400 $\mathrm{m}$ sur $1400 \mathrm{~m}$, uniforme dans la direction longshore (un profil central reproduit de part et d'autre). La zone d'étude s'étend donc du sommet de la dune jusqu'à -10 m (CM) dans le chenal qui sépare le Banc Hills de la plage. Le tableau 1 présente les données à fournir en entrée des 3 modèles. Pour SBEACH, un seul profil cross-shore de $1400 \mathrm{~m}$ est utilisé. Les données qui servent à valider et calibrer les modèles sont issues de campagnes de mesure du LOG effectuées en Février (par temps calme) puis Novembre 2007 (tempête) : des profils topographiques dune-plage complétés par des données GEBCO jusqu'à $-10 \mathrm{~m}$. Les conditions de forçage appliquées aux limites des domaines sont des données horaires de niveau d'eau (source : SONEL) et de paramètres de vagues (issues de la propagation SWAN). Pour SBEACH, les conditions de vent au large (données Météofrance) sont également appliquées. Le maillage de calcul choisi pour le code X-BEACH est une grille uniforme de $5 \mathrm{~m}$ cross-shore sur $200 \mathrm{~m}$ longshore, et pour TELEMAC des maillages de 10, 20 et $50 \mathrm{~m}$ uniformes dans les deux directions donnent des résultats équivalents. Pour SBEACH une maille fine de $2 \mathrm{~m}$ a été employée le long du profil. Des mesures hydrodynamiques in-situ ont été réalisées dans le profil central du domaine, et les résultats des simulations seront extraits sur ce profil.

\section{Résultats préliminaires et discussion}

Les codes X-BEACH et TELEMAC ont été paramétrés pour deux périodes de validation: par "temps calme" en février 2007 et "tempête" en novembre 2007. 
SBEACH reproduisant très mal les conditions de faibles houles (SABATIER et al., 2002) et se limitant à la modélisation épisodique des impacts des tempêtes sur les profils, sa validation a été réalisée à partir d'évènements tempétueux plus anciens. Ne seront présentés ici que les résultats issus des simulations "tempête".

\subsection{Validation hydrodynamique}

Pour évaluer la qualité des résultats hydrodynamiques, la tempête du 9 novembre 2007 a été choisie comme période de validation de tempête. Un appareil de mesures Midas Valeport, enregistrant à $15 \mathrm{~cm}$ du fond les vitesses et direction du courant, a été déployé à $+1,85 \mathrm{~m}(\mathrm{CM})$ sur l'estran lors de cet évènement de forte houle associé à un coup de vent de Nord. Une surcote record de 2,40 m à basse mer (période de retour $>50$ ans) a été enregistrée le 9 novembre à Dunkerque lors de cet évènement (MASPATAUD, en cours).

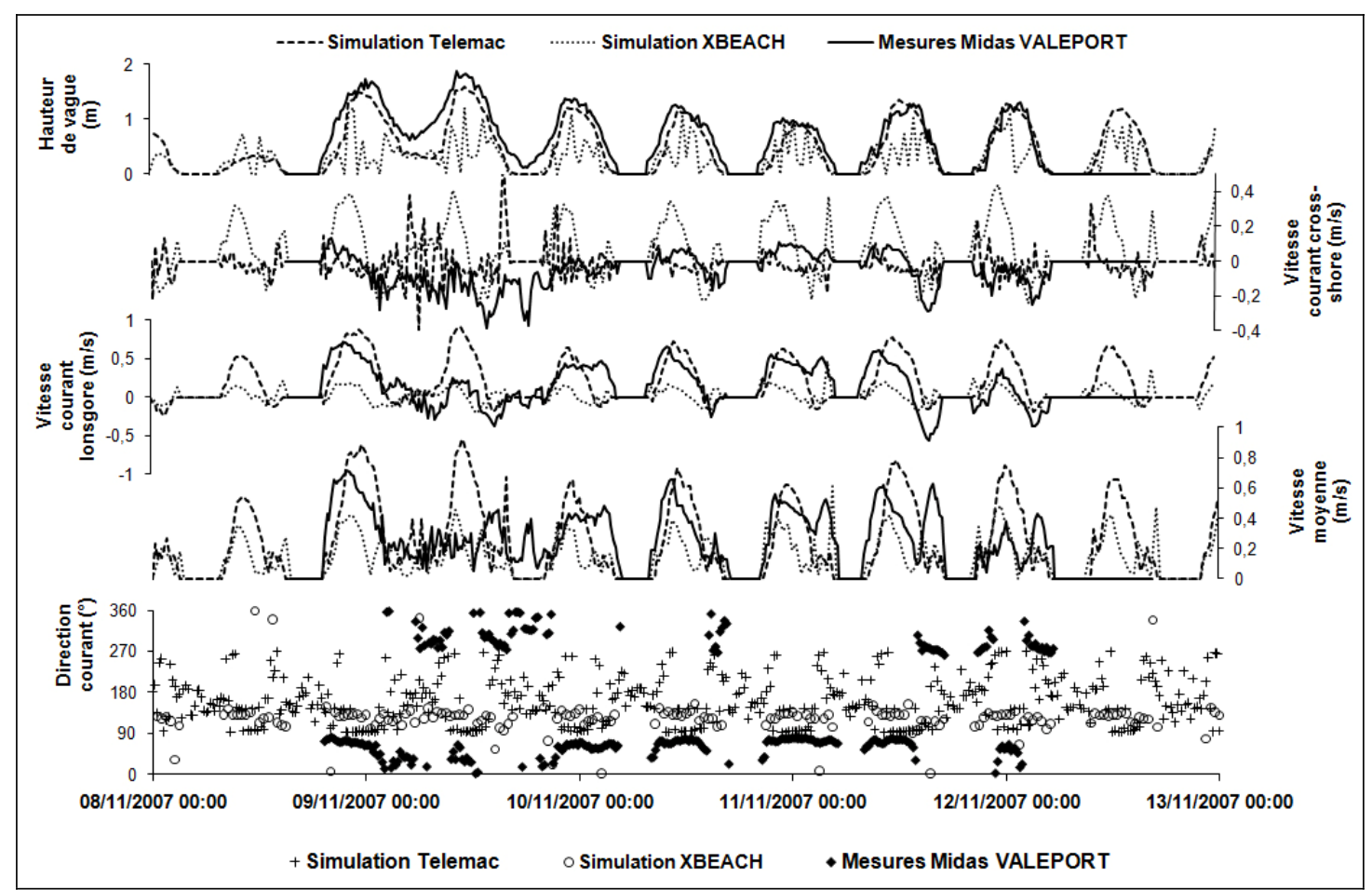

Figure 2. Comparaison des hauteurs de vague, vitesses cross-shore et longshore, vitesse et direction moyenne du courant, modélisées et mesurées in-situ - Tempête de novembre 2007.

La figure 2 présente les premiers résultats des simulations réalisées sur la période de tempête du 8 au 13 novembre 2007. Les données modélisées donnent des résultats proches de la réalité en termes de hauteur de vagues, en particulier la chaine TELEMAC qui reproduit bien les fortes vagues mesurées sur l'estran par le capteur dans la journée du 9 novembre. En termes de circulation hydrodynamique, les vitesses des courants 
longshore modélisés par XBEACH sont légèrement sous-estimées par rapport aux mesures in-situ. Mais l'asymétrie de ces courants longitudinaux est plutôt bien restituée avec une dominance du flot vers le Nord-Est. L'amplitude des vitesses de courant crossshore est en revanche mieux restituée avec TELEMAC, sauf sur la période de forte agitation du 9 novembre. Le constat est similaire pour la vitesse moyenne du courant où la simulation XBEACH sous-estime tandis que TELEMAC surestime les valeurs réelles enregistrées au Valeport. Les écarts obtenus entre les valeurs calculées et mesurées peuvent s'expliquer en partie par le fait que les simulations ont été réalisées sur une bathymétrie uniforme alors que la bathymétrie réelle comporte des irrégularités le long de l'axe longshore pouvant engendrer des circulations locales. Une lacune qui pourra être comblée ultérieurement grâce à des mesures topographiques plus étendues.

L'intensité et la direction des courants durant la tempête ont été plus difficilement reproduites par les modèles. En effet, dans ces conditions très énergétiques d'une houle frontale, les courants de marée ne sont plus prédominants, car l'apport important de masse d'eau par les vagues à la côte, provoque en compensation un fort courant de retour dirigé vers le large, facteur de l'évolution du profil des plages. Le forçage dû au vent aggrave certainement aussi l'impact des vagues à la côte.

\subsection{Validation morphodynamique}

La chaine de calcul Telemac, qui intègre le module sédimentaire Sisyphe, ne prend pas en compte la falaise dunaire et montre des difficultés à reproduire le déplacement crossshore des barres intertidales dans le cas où les courants de marée longshore sont importants. Plusieurs essais de formules de transport (telles qu'elles sont modélisées dans Sisyphe) donnent pour l'instant les mêmes résultats avec Bijker, Bijker et prise en compte de la houle, Van Rijn et Soulsby-Van Rijn. Les variations morphologiques des barres-bâches, à l'échelle centimétrique ici, ne seront pas représentées dans la figure de synthèse.

La figure 3 présente donc les évolutions morphologiques mesurées et modélisées dans le profil cross-shore central du domaine pour SBEACH et XBEACH. Les modèles génèrent un lissage de l'estran (assez léger pour XBEACH, total pour SBEACH) avec un comblement des bâches et une érosion des barres. Accusant une petite perte d'information au niveau de la dune avec le maillage de $5 \mathrm{~m}$, le code XBEACH estime l'abaissement du pied de dune à $0,30 \mathrm{~m}$, mais sans aucun recul du front dunaire. En revanche, le modèle $\mathrm{SBEACH}$, qui néglige pourtant les transports longshore de sédiments induits par les courants, reproduit très fidèlement le recul de la falaise sableuse d'au minimum $1 \mathrm{~m}$. Le maillage plus fin employé avec SBEACH pourrait expliquer le bon rendu morphologique de l'érosion induite par cette tempête. Lors de la simulation, le recul du pied de dune se produisant uniquement lors des plus hautes marées, une durée de simulation plus courte permettrait d'obtenir des sorties plus proches de la réalité. 


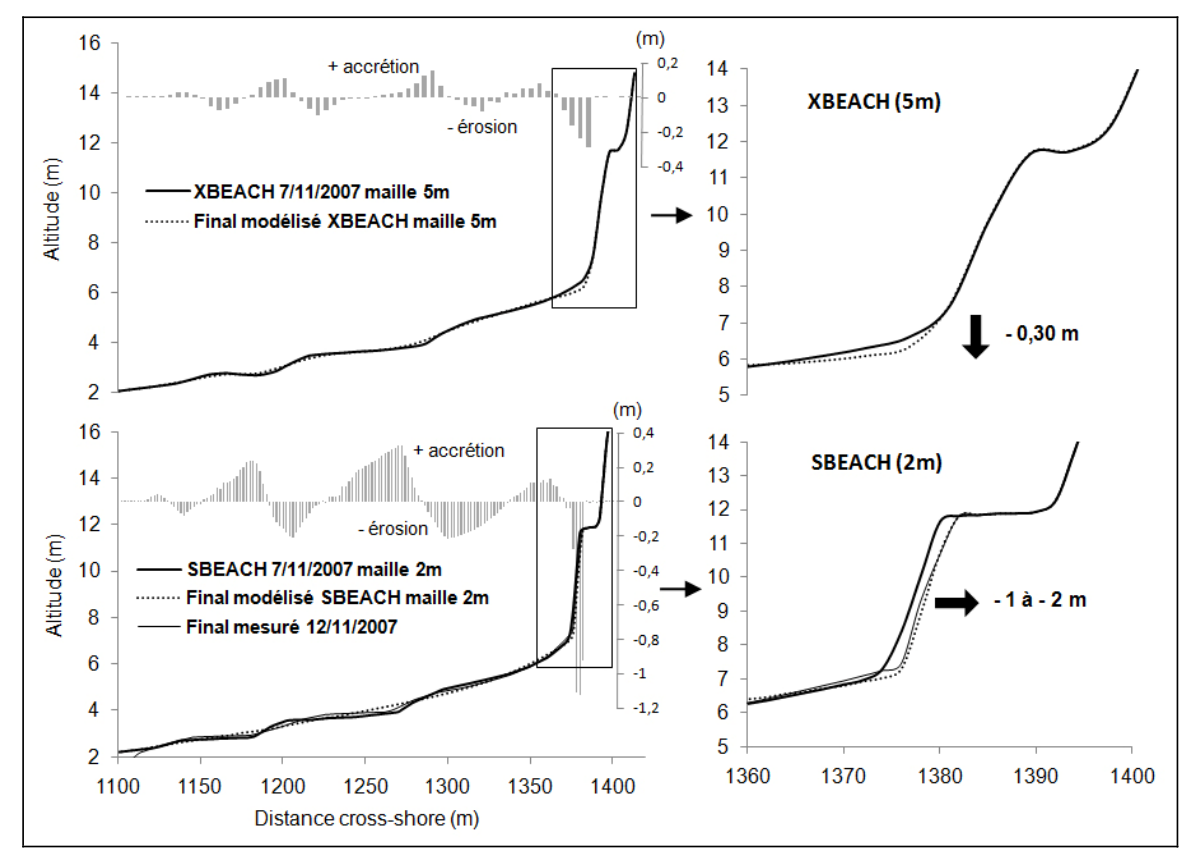

Figure 3. Résultats des simulations morphodynamiques issues des modèles : profils cross-shore, accrétion/érosion modélisée, agrandissement centré sur la falaise dunaire.

\section{Conclusion}

La comparaison avec les observations permet d'estimer les effets d'une houle de tempête superposés au courant de marée, en milieu macrotidal, sur une portion de littoral en érosion. Dans leur état actuel, aucun des trois modèles mis en place sur la plage de la Dune Dewulf ne restitue à lui seul les mêmes conditions hydrodynamiques ni ne reproduit exactement le recul dunaire tel qu'il a été mesuré in situ. Pourtant, ces codes sont tous pertinents pour étudier l'impact des conditions extrêmes sur le profil dune-plage. Cette étude met ainsi en évidence la complémentarité des 3 modèles utilisés ainsi que la nécessité de continuer à affiner leurs implantations sur le site d'étude, pour tenir compte de l'ensemble des processus en jeu, dans le cas de conditions topographiques complexes et hydrodynamiques extrêmes, à partir de données in situ.

Remerciements : Ces travaux ont été en partie financés par l'Agence Nationale de la Recherche (ANR) au travers du programme VMC (projet VULSACO, $\mathrm{n}^{\circ}$ ANR VMC06-009). A. Maspataud bénéficie d'une Allocation de recherche du Ministère de l'Enseignement et de la Recherche.

\section{Références bibliographiques}

BAART F., VAN DER KAAIJ T., VAN ORMLONDT M., VAN DONGEREN A., VAN KONINGSVELD M., ROELVINK J.A., (2009) Real-time forecasting of morphological storm impacts: a case study in the Netherlands, Journal of Coastal Research SI 56, ICS2009, pp 1617-1621. 
Thème 2 -Dynamique sédimentaire et transports des particules

BOOIJ N., HAAGSMA IJ.G., HOLTHUIJSEN L.H., KIEFTENBURG A.T.M.M., RIS R.C., VAN DER WESTHUYSEN A.J., ZIJLEMA M. (2004). Swan Cycle III version 40.41. User's Manual, $115 \mathrm{p}$.

CHARLES E. (2008). Modélisation morphodynamique côtière : morphodynamique court-terme de la plage de Zuydcoote. Rapport final, BRGM/ARN/ESL - projet VULSACO, $100 \mathrm{p}$.

HEQUETTE A., RUZ M.H., MASPATAUD A., SIPKA V. (2009). Effects of nearshore sand bank and associated channel on beach hydrodynamics: implications for beach and shoreline evolution. Journal of Coastal Research SI 56, ICS2009, pp 59-63.

HERVOUET J.M. (2007). Hydrodynamics of Free Surface Flows: Modelling with the Finite Element Method. John Wiley \& Sons., 360 p. doi:10.1002/9780470319628

LARROUDE P. (2008). Methodology of seasonal morphological modelisation for nourishment strategies on a Mediterranean beach. Marine Pollution Bulletin 57, pp 47-52. doi:10.1016/j.marpolbul.2008.04.039

LARSON M., KRAUS N.C. (1989). SBEACH: numerical model for simulating storminduced beach change. Report 1, empirical fondation and model development. CERCWES, Techn. Report CERC-89-9.

MASPATAUD A. (en cours). Impacts des tempêtes sur la morphodynamique du profil côtier en milieu macrotidal. Thèse, Université du Littoral Côte d'Opale.

MORELlATO D., SABATIER F., PONS F., GAUFRES P. (2004). Comparaison de deux modèles cross-shore d'érosion des plages (Site de Rousty, Delta du Rhône, France). VIIIèmes Journées Nationales Génie Civil - Génie Côtier, Compiègne, 7-9 septembre 2004, pp 203-210. doi:10.5150/jngcgc.2004.023-M

ROELVINK D., RENIERS A., VAN DONGEREN A., VAN THIEL DE VRIES J., LESCINCKI J., McCALL R. (2008). Modelling hurricane impacts on beaches, dunes and barrier islands. Unesco-IHE Institute for Water Education, Deltares and Delft University of Technology, $30 \mathrm{p}$.

RUZ M-H., HEQUETTE A., MASPATAUD A. (2009). Identifying forcing conditions responsible for foredune erosion on the northern coast of France. Journal of Coastal Research, SI 56, ICS2009, pp 356-360.

SABATIER F. (2008). Modélisation de l'impact du changement climatique sur l'érosion des dunes. Application à la Camargue. La Houille Blanche, 1, pp 40-49. doi:10.1051/lhb:2008004

SABATIER F., LAMBERT A., CHAIBI M., SAMAT O., PROVANSAL M. (2002). Morphodynamique du profil de plage en milieu microtidal : du relevé de terrain au modèle mathématique et numérique. In : "Geomorphology: from expert opinion to modelling", Strasbourg, 26-27 avril, D. Delahaye, F. Levoy, O. Maquaire, pp 357-360. VILLARET C. (2004). Modélisation du transport littoral. VIIIèmes Journées Nationales Génie Civil - Génie Côtier, Compiègne, 7-9 septembre 2004, pp 251-260. DOI:10.5150/jngcgc.2004.028-V 